\title{
Fingertip force and electromyography of finger flexor muscles during a prolonged intermittent exercise in elite climbers and sedentary individuals
}

\author{
LAURENT VIGOUROUX \& FRANCK QUAINE
}

EA 597, UFR APS, Université foseph Fourier, Grenoble, France

(Accepted 29 November 2004)

\begin{abstract}
The aim of this study was to characterize forearm muscle fatigue identified by the decrease in electromyogram median frequency and/or fingertip force during intermittent exercise. Nine elite climbers (international competitive level, USA 5.14a on sight) and ten non-climbers were instructed to maintain a fingertip force of $80 \%$ of their maximal voluntary contraction force on a dynamometer mimicking a rock climbing grip during a $5 \mathrm{~s}$ effort $/ 5 \mathrm{~s}$ rest cycle for 36 repetitions (i.e. 6 min of exercise). Elite climbers lasted twice as long as non-climbers (climbers: $3 \mathrm{~min}$; non-climbers: $1 \mathrm{~min} 30 \mathrm{~s}$ ) before the force could no longer be maintained (i.e. the failure point). After this moment, fingertip force decreased and stabilized until the end of the exercise around 50\% maximum voluntary contraction force in non-climbers and $63 \%$ in elite climbers. Electromyogram median frequency showed a greater decrease in non-climbers than in elite climbers before the failure point. No change in median frequency was observed after the failure point in elite climbers or in non-climbers. These results confirm that elite climbers are better adapted than non-climbers for performing the intermittent fingertip effort before the failure point. After this point, the better fingertip force of elite climbers suggests different forearm muscle properties, while the electromyography results do not provide any indication about the fatigue process.
\end{abstract}

Keywords: Rock climbing, electromyography, finger flexor muscles, fatigue

\section{Introduction}

Over the last 20 years, interest in sport climbing has increased considerably and it is now an international competitive sport (Watts \& Drobish, 1998). It involves climbing walls by gripping holds with the fingers of each hand in succession. This effort corresponds to an intermittent exercise of the forearm muscles (Ferguson \& Brown, 1997). The inability to generate and/or to sustain the finger force necessary to maintain contact with the hold is the main reason for an unsuccessful ascent or failure on a given climbing move (Watts, Dagget, Gallagher, \& Wilkins, 2000). Fatigue has been defined by Enoka and Stuart (1992) as an acute impairment of performance that includes both an increase in the perceived effort necessary to exert a desired force and the eventual inability to produce this force. Fatigue of the forearm muscles is critical in rock climbing, since it can decrease the intensity of the force applied on the hold.
Fatigue can be detected before any force modification by monitoring changes in the surface electromyogram (EMG) of contracting muscles. It has been shown that the EMG median frequency is a reliable index of fatigue within a muscle (Basmajian \& De Luca, 1985; Petrofsky, 1981). The EMG median frequency is related to the time course of the fatiguelinked physiological and biochemical processes early in the contraction. During sustained contractions, the decrease in EMG median frequency is causally related to the decrease in conduction velocity of motor potentials on the sarcolemma. This is mostly due to the decrease in the $\mathrm{pH}$ of the interstitial fluid, as lactic acid and hydrogen and potasium ions $\left(\mathrm{H}^{+}\right.$ and $\mathrm{K}^{+}$) accumulate during the contraction, with the casual influence of $\mathrm{H}^{+}$having been more widely studied (Bigland-Ritchie, Donovan, \& Roussos, 1981; Enoka \& Stuart, 1992; Hagg, 1992; Petrofsky, 1981; Sjogaard, Savard, \& Juel, 1988).

The EMG median frequency of forearm muscles was successfully used to examine fatigue during 
simulated rock climbing grips (Quaine \& Vigouroux, 2004). Participants were required to sustain intermittent fingertip force at $80 \%$ of their maximal voluntary contraction (MVC) force until it could no longer be maintained (i.e. the failure point). The EMG median frequency decreased twice as fast for non-climbers than elite climbers. Thus elite climbers demonstrated physiological and biochemical adaptations compared with non-climbers. Pitcher and Miles (1997) showed that when non-climbers prolonged exercise after the failure point, the force intensity fell steadily to reach a plateau at approximately 50\% MVC. In comparison, elite climbers may be expected to present a different pattern.

The aim of the current study was to characterize forearm fatigue identified by the decrease in EMG median frequency and/or fingertip force during intermittent hand grip exercise in both elite climbers and non-climbers.

\section{Methods}

Nine elite climbers (international competitive level, USA $5.14 \mathrm{a}$ on sight; age $22.2 \pm 1.6$ years, height $1.76 \pm 0.04 \mathrm{~m}$, body mass $65.5 \pm 3.2 \mathrm{~kg}$; mean $\pm s$ ) and ten non-climbers who had not practized forearm contractions during sports or work (age $24.0 \pm 1.8$ years, height $1.78 \pm 0.05 \mathrm{~m}$, body mass $74.0 \pm$ $3.0 \mathrm{~kg}$ ) took part in the study. All participants were right-handed males. They signed an informed consent in accordance with the guidelines of Joseph Fourier University.

\section{Apparatus}

The finger-grip design described by Quaine, Vigouroux and Martin (2003) was used in this study

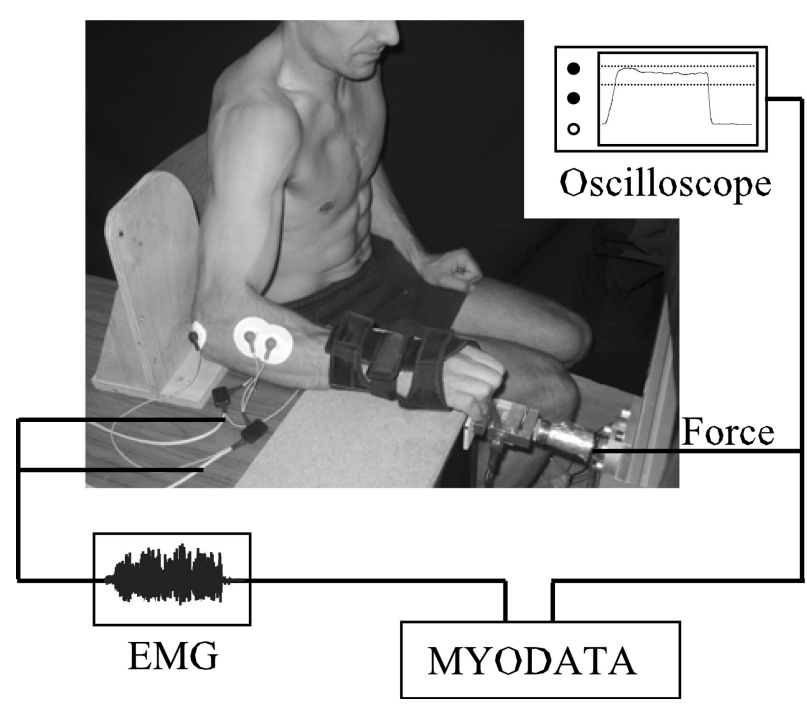

Figure 1. The experimental set-up.
(Figure 1). This apparatus allowed us to evaluate precisely the force exerted at the participant's fingertips. The participants were seated with their right forearm placed in the finger-grip. The shoulder position was standardized at $45^{\circ}$ of abduction and the elbow was flexed $90^{\circ}$.

The most common finger posture used by up to $90 \%$ of sport climbers (Schweizer, 2001), the crimp grip, was examined. The distal interphalangeal joint was hyper-extended $\left(-30 \pm 9^{\circ}\right)$ and the proximal interphalangeal joint was flexed $\left(90 \pm 16^{\circ}\right)$. The joint angles were controlled in the sagittal plane using a digital camera (Sony, DSC-S70, Japan). The fingers gripped the hold on a surface $1 \mathrm{~cm}$ deep. The wrist was held thanks to a mitten (Salomon, Model Protege-Poignet Active Men, France) at $40^{\circ}$ of extension in front of the hold. The thumb did not act as an additional gripping force. A mono-axial load cell (Schlumberger, model CD-750, France) was used to measure the grip force intensity applied by the fingers. The signal was amplified (PM, model 1965, France) before recording. An oscilloscope provided the participants with constant visual feedback of the force produced and indicated the target force of $80 \%$ of their MVC on the screen.

\section{Electromyography}

Surface EMG activity of the extrinsic hand flexor muscles - that is, the flexor digitorum superficialis (FDS) and flexor digitorum profundus (FDP) - was recorded. Extensor muscle EMG from the extensor digitorum communis (EDC) was also recorded to control the EDC involvement during the exercise. As reported previously (Quaine et al., 2003), the ratio of flexor and extensor EMG amplitude was constant throughout the exercise in both groups of participants, suggesting a constant co-activation. Preamplifier $(\times 600)$ Ag-AgCl electrodes (Graphic Controls, Canada) were used (input impedance 10 $\mathrm{G} \Omega$, common mode rejection ratio $=100 \mathrm{db}$ at 50 / $60 \mathrm{~Hz}$, bandwidth $=5-1000 \mathrm{~Hz}$ ). The surface electrodes were placed as suggested by Quaine et al. (2003). To reduce the influence of the flexor carpi radialis (i.e. a wrist flexor), the position of the finger flexor muscle electrodes was considered appropriate when the EMG signal was present during finger flexion with a stable wrist, yet absent during wrist flexion with no voluntary finger flexion (Blackwell, Kornatz, \& Heath, 1999). The electrodes for the extensor muscles were positioned around the $\frac{1}{4}$ point on a line drawn from the lateral epicondyle to the styloid process of the ulna (Basmajian \& De Luca, 1985). Force and EMG signals were sampled at $1024 \mathrm{~Hz}$ (Mazet Electronique, model Biostim 6082, France). The raw EMG data were filtered using a 
low-pass filter $(300 \mathrm{~Hz}$ cut-off, zero lag fourth-order Butterworth filter).

\section{Maximal voluntary contraction}

The participants warmed up on the device by performing a series of 100 contractions for $5 \mathrm{~s}$ at $50 \mathrm{~N}$ as advised by Schweizer (2001). The EMG parameters collected during the warm-up showed that no fatigue was produced. After a $3 \mathrm{~min}$ rest, the participants performed three maximal isometric finger flexion contractions for $5 \mathrm{~s}$ separated by a 5 min rest period. The highest peak force was chosen as the pre-exercise maximal voluntary contraction (MVC) force.

\section{Intermittent exercise}

After a 10 min rest, the participants performed finger flexion contractions at $80 \% \mathrm{MVC}$ for $5 \mathrm{~s}$ followed by $5 \mathrm{~s}$ of rest. During the rest period, they could relax, but were not allowed to release the fingers from the hold and were instructed to keep the arm and the hand in the same posture. This protocol is appropriate to study climbers' forearm muscle fatigue, since it mimics the forearm exercise encountered during rock-climbing (Pitcher \& Miles, 1997; Quaine et al., 2003). When the participants were no longer able to maintain $80 \%$ of pre-exercise MVC, they were required to exert the highest voluntary contractions they could perform. The exercise consisted of 36 repetitions, which took 6 min to complete. Oral encouragement was given to each participant to obtain maximal effort during both the maximal voluntary contractions and intermittent exercise.

\section{Data analysis}

Each contraction was labelled from 1 to 36 (i.e. $\mathrm{C}_{1}$ to $\mathrm{C}_{36}$ ). For each contraction, fingertip force and EMG were divided into $2 \mathrm{~s}$ windows of data with the onset of analysis adjusted $2 \mathrm{~s}$ after the force started to increase. The moment the required force could no longer be maintained (i.e. the failure point) was determined for each participant using a thresholdbased criterion set to $80 \pm 10 \%$ MVC and labelled $\mathrm{C}_{\mathrm{TF}}$ (Quaine et al., 2003). For each of the two groups of participants, $\mathrm{C}_{\mathrm{TF}}$ was averaged and expressed in seconds (i.e one contraction corresponds to $10 \mathrm{~s}$ of exercise, $5 \mathrm{~s}$ contraction and $5 \mathrm{~s}$ rest).

The median frequency $\left(f_{\text {med }}\right)$ was computed after a fast Fourier transform for each $2 \mathrm{~s}$ segment (Basmajian \& De Luca, 1985). Both fingertip force and $f_{\text {med }}$ were normalized according to their values recorded during the MVC. All computation procedures were performed using Matlab software (The Math Works, Inc.,USA).

Descriptive statistics are reported as the mean \pm standard deviation in the text and the mean \pm standard error of the mean in the figures. Cochran's test for homogeneity of variance was used to assess the normality of both the force and EMG data. An independent $t$-test was used to compare fingertip force MVC between non-climbers and elite climbers. Two-way analyses of variance (group $\times$ contraction number) with repeated measures on the second factor were used to analyse the fingertip force and median frequency. The mean time-related behaviour of $f_{\text {med }}$ for the flexor muscles was computed for each of the two groups. The slopes of best fit were plotted from $\mathrm{C}_{1}$ to $\mathrm{C}_{\mathrm{TF}}$ and from $\mathrm{C}_{\mathrm{TF}}$ to $\mathrm{C}_{36}$. Significance was set at $P<0.05$ for all statistical tests.

\section{Results}

Cochran's test of homogeneity showed that both fingertip force and median frequency were normally distributed $(P>0.05)$. Technical problems meant that the EMG of one non-climber did not provide satisfactory signals. Thus the EMG data of this participant were removed from the statistical analyses.

\section{Maximal voluntary contractions}

The mean MVC force of the elite climbers was $412.3 \pm 40.9 \mathrm{~N}$ and that of the non-climbers was $361.6 \pm 52.1 \mathrm{~N}$. The $t$-test $(t=-2.33, P<0.05)$ showed that the force exerted by the non-climbers was significantly lower than that exerted by the elite climbers.

\section{Intermittent exercise}

The results for fingertip force in both elite climbers and non-climbers are presented in Figure 2. The analysis showed a significant main effect of group $\left(F_{1,17}=9.122, P<0.05\right)$. This means that the climbers applied significantly higher overall fingertip forces than non-climbers (climbers: $67.6 \pm 5.9 \%$ MVC; non-climbers: $56.2 \pm 12.9 \%$ MVC). The analysis of variance (ANOVA) showed a significant main effect of contraction number $\left(F_{35,595}=39.63\right.$, $P<0.05)$. This means that overall fingertip force (averaged over the two groups) was significantly different between contractions. A significant group $\times$ contraction number interaction $\left(F_{35,595}=6.93, P\right.$ $<0.05$ ) was observed. This shows that the fingertip force applied by elite climbers and non-climbers evolved differently throughout the exercise. 
The mean contraction number corresponding to the failure point (i.e. $\mathrm{C}_{\mathrm{TF}}$ ) was $9.2 \pm 4.5$ contractions for the non-climbers and $19.0 \pm 13.6$ contractions for the elite climbers. These corresponded to $1 \mathrm{~min} 30 \mathrm{~s}$ for non-climbers and $3 \mathrm{~min}$ for elite climbers. After $\mathrm{C}_{\mathrm{TF}}$, fingertip force decreased significantly and reached $63.2 \pm 1.6 \% \mathrm{MVC}$ for elite climbers and $50.0 \pm 7.8 \%$ MVC for nonclimbers.

The variations in median frequency $\left(f_{\text {med }}\right)$ are presented in Figure 3. The statistical analysis showed a significant main effect of group $\left(F_{1,16}=5.59\right.$, $P<0.05)$, meaning that the overall $f_{\text {med }}$ was different

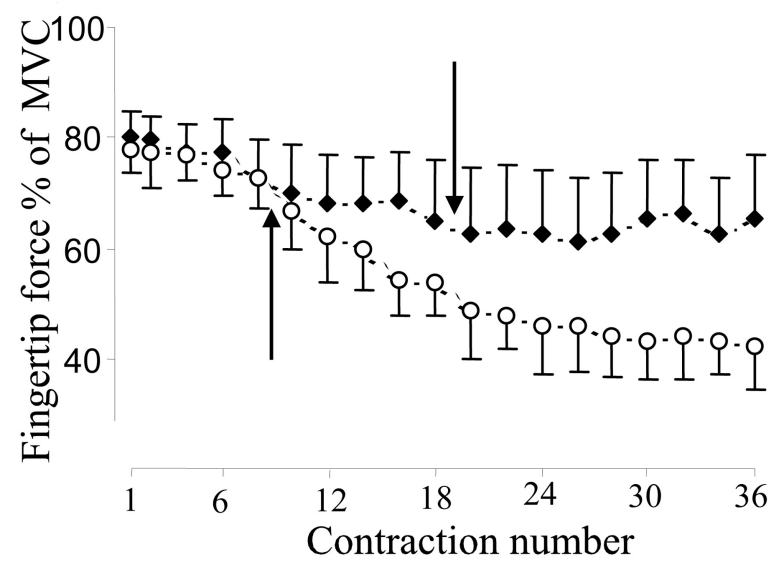

Figure 2. Mean ( \pm standard error of the mean) normalized fingertip force in elite climbers $(\diamond)$ and non-climbers $(O)$ during the intermittent exercise (36 contractions). To improve clarity, only every second contraction is represented. The arrows show the moment at which the initial force (i.e. $80 \pm 10 \% \mathrm{MVC}$ ) could no longer be maintained (i.e. the failure point, $\mathrm{C}_{\mathrm{TF}}$ ).

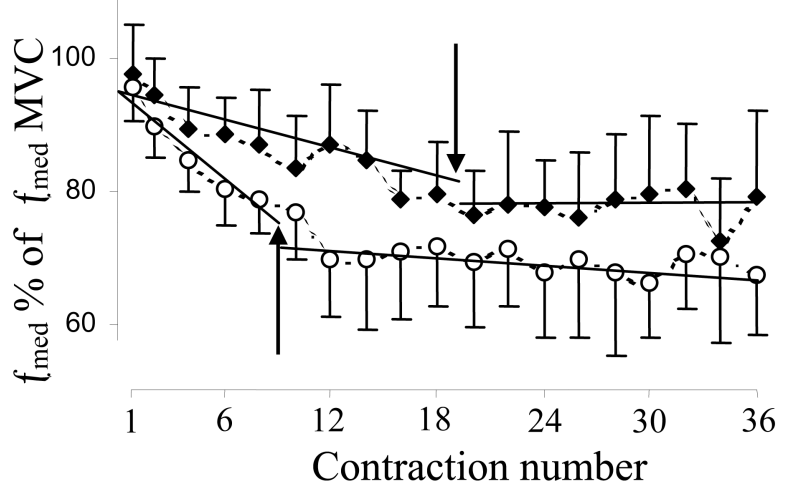

Figure 3. Mean ( \pm standard error of the mean) normalized median frequency $\left(f_{\text {med }}\right)$ in elite climbers $(\diamond)$ and non-climbers (O) during the intermittent exercise (36 contractions). The arrows show the moment at which the initial force (i.e. $80 \pm 10 \% \mathrm{MVC}$ ) could no longer be maintained (i.e. the failure point, $\mathrm{C}_{\mathrm{TF}}$ ). Lines of best fit were plotted from $\mathrm{C}_{1}$ to $\mathrm{C}_{\mathrm{TF}}$ and from $\mathrm{C}_{\mathrm{TF}}$ to $\mathrm{C}_{36}$. To improve clarity, only every other contraction is presented. for elite climbers and non-climbers (non-climbers: $73.5 \pm 7.3 \% f_{\text {med }}$ MVC; climbers: $82.3 \pm 6.3 \% f_{\text {med }}$ MVC). A significant main effect of contraction number $\left(F_{35,560}=10.08, P<0.05\right)$ demonstrated that the overall $f_{\text {med }}$ (averaged over the two groups) was significantly different between contractions. The ANOVA revealed a non-significant group $\times$ contraction number interaction, indicating that $f_{\text {med }}$ evolved similarly throughout the exercise in both groups.

From $\mathrm{C}_{1}$ to $\mathrm{C}_{\mathrm{TF}}$, the $f_{\text {med }}$ slopes were negative in elite climbers and non-climbers. A steep slope was observed for non-climbers $(y=-2.34 x+95.9$, $r=0.96)$, whereas that for elite climbers $(y=-0.9 x+95.6, r=0.89)$ was around $2 \frac{1}{2}$ times less steep. From $\mathrm{C}_{\mathrm{TF}}$ to the end of the exercise $\left(\mathrm{C}_{36}\right)$, the slope amounted to -0.05 in elite climbers $(y=-0.05 x+78, r=0.14)$ and to -0.14 in nonclimbers $(y=-0.14 x+72, r=0.44)$. This indicates that $f_{\text {med }}$ did not evolve on average after the moment the initial contraction could no longer be maintained (i.e. the failure point). From $\mathrm{C}_{\mathrm{TF}}$ to $\mathrm{C}_{36}$, the mean $f_{\text {med }}$ amounted to $69.2 \pm 2.1 \% f_{\text {med }}$ MVC for non-climbers and $77.3 \pm 2.1 \% f_{\text {med }}$ MVC for elite climbers.

\section{Discussion}

Our results indicate that the maximal voluntary fingertip force applied by elite climbers is significantly greater than that applied by non-climbers. Similar results are presented in the literature, showing that climbers have developed their fingertip force capacity, enabling them to maintain contact with the hold during rock climbing (Cutts \& Bollen, 1993. Grant, Hynes, Whittaker, \& Aitchison, 1996). Assessing the failure point at which this contraction can no longer be maintained shows that elite climbers sustain the predetermined force twice as long as non-climbers. The failure point is thus useful for detecting fatigue in rock climbing (Watts, Newbury, \& Sulentic, 1996), but it does have its limitations. The failure point is a function of both physiological and psychological factors (Enoka \& Stuart, 1992). Non-climbers could thus be less motivated, since they are less accustomed than elite climbers to the pain and general discomfort associated with finger-grip tasks (Janot, Steffen, Porcari, \& Maher, 2000. Pandolf, Kamon, \& Noble, 1978). However, these may be overcome by using EMG data. The EMG spectral modifications provide unambiguous evidence about the rate of the fatigue process. Before the failure point, the EMG median frequency decreases in elite climbers and in nonclimbers. These decreases show that there is a fatigue progression concomitant with the accumulation of interstitial biochemical by-products. When the blood 
flow is occluded (i.e. for contractions higher than $30 \%$ MVC), the only known factor that affects the conduction velocity is the amount of interstitial $\mathrm{H}^{+}$ and $\mathrm{K}^{+}$(Bigland-Ritchie et al., 1981; Enoka \& Stuart, 1992; Hagg, 1992; Petrofsky, 1981; Sjogaard et al., 1988).

This accumulation is lower in elite climbers, since the median frequency decreases 2.5 times more slowly than in non-climbers. One explanation could be found in the studies of Ferguson and Brown (1997) and Usaj (2002), who showed that elite climbers show superior peripheral vascular characteristics and enhanced vasodilator capacities. Hence, the blood flow between each contraction is increased in elite climbers, which determines the rate at which biochemical by-products are removed.

After the failure point, no EMG median frequency changes were observed in elite or non-climbers, suggesting that no biochemical modifications occur in the muscles. During this phase, fingertip force evolved differently between the groups. In nonclimbers, the force fell until a second plateau was reached at 50\% MVC. Similar results have been described for non-climbers by Pitcher and Miles (1997). These authors concluded that only the slowtwitch oxidative fibres (Type I) were at work after the failure point. In elite climbers, the force failure was more limited and stabilized around 60\% MVC. This result is new and suggests that elite climbers have adapted forearm muscle capacities to apply a high intensity of force to the hold despite the fatigue process. However, it cannot be established by the present study, or by reports in the literature, whether the maintenance of this level of force was attributable to slow-twitch oxidative fibres as in non-climbers. Regarding force intensity, elite climbers may experience different forearm muscle fibre recruitment and/ or composition resulting in the maintenance of a higher intensity of force after the failure point. This is in line with the fibre-based muscle model of Hawkins and Hull (1993). These authors proposed a model for predicting muscle fatigue from muscle fibre composition. The development of forearm muscle fatigue in the elite climbers in the present study suggests fatigue of an equivalent heterogeneous muscle composed of all three fibre types (i.e. slow-twitch oxidative, fasttwitch glycolytic oxidative and fast-twitch glycolytic fibres). Additional experiments will need to be performed to confirm this assumption.

Within the limits of the current study, the experimental protocol allows a broadening of our understanding of the patterns and mechanisms of the development of fatigue during simulated rock climbing. Applications include the monitoring of training programmes, particularly the adaptations observed in the forearm muscles before the failure point.

\section{Conclusions}

During a prolonged intermittent finger grip exercise, forearm muscle electromyograms show a greater decrease in median frequency in non-climbers than in elite climbers before the failure point, suggesting a greater rate of fatigue. After the failure point, no changes in frequency are observed in elite climbers or non-climbers, suggesting no biochemical changes in the muscles. Elite climbers are thus able to maintain forceful fingertip force longer than nonclimbers. They limit the force loss induced by fatigue and maintain a higher percentage of force intensity after the moment the initial target force can no longer be maintained.

\section{References}

Basmajian, J. V., \& De Luca, C. J. (1985). Muscles alive. Baltimore, $\mathrm{MD}$ : Williams \& Wilkins.

Bigland-Ritchie, B., Donovan, E. F., \& Roussos, C. S. (1981). Conduction velocity and EMG power spectrum changes in fatigue of sustained maximal efforts. Fournal of Applied Physiology, 51, 1300-1305.

Blackwell, J. R., Kornatz, K. W., \& Heath, M. E. (1999). Effect of grip span on maximal grip force and fatigue of flexor digitorum superficialis. Applied Ergonomics, 30, 401-405.

Cutts, A., \& Bollen, R. (1993). Grip strength and endurance in rock climbers. Proceedings of the Institute of Mechanical Engineers, 207, $87-92$

Enoka R. M., \& Stuart D. G. (1992). Neurobiology of muscle fatigue. Fournal of Applied Physiology, 72, 1631-1648.

Ferguson, R. A., \& Brown, M. D. (1997). Arterial blood pressure and forearm vascular conductance responses to sustained and rhythmic isometric exercise and arterial occlusion in trained rock climbers and untrained sedentary subjects. European fournal of Applied Physiology, 76, 174-180.

Grant, S., Hynes, V., Whittaker, A., \& Aitchison, T. (1996). Anthropometric, strength, endurance, and flexibility characteristics of elite and recreational climbers. Fournal of Sports Sciences, 14, 301-309.

Hagg, C. (1992). Interpretation of EMG spectral alterations and alteration indexes at sustained contraction. Fournal of Applied Physiology, 73, 1211-1217.

Hawkins, D., \& Hull, M. L. (1993). Muscle force as affected by fatigue: Mathematical model and experimental verification. Fournal of Biomechanics, 26, 1117-1128.

Janot, J., Steffen, J., Porcari, J., \& Maher, M. (2000). Heart rate responses and perceived exertion for beginner and recreational sport climbers during indoor climbing. Fournal of Exercise Physiology, 3, 1-7.

Pandolf, K., Kamon, E., \& Noble, B. (1978). Perceived exertion and physiological reponses during negative and positive work in climbing a laddermill. Fournal of Sport Medicine and Physical Fitness, 18, 227-236.

Petrofsky, J. S. (1981). Quantification through the surface EMG of muscle fatigue and recovery during successive isometric contractions. Aviation, Space and Environmental Medicine, 52, $545-550$.

Pitcher, J. B., \& Miles, T. S. (1997). Influence of muscle blood flow on fatigue during intermittent human hand-grip exercise and recovery. Clinical and Experimental Pharmacology and Physiology, 24, 471-476. 
Quaine, F., \& Vigouroux, L. (2004). Effect of simulated rock climbing grips on maximal fingertip force and fatigue of the extrinsic muscle of the hand. International fournal of Sport Medicine, 25, 634-637.

Quaine, F., Vigouroux, L., \& Martin, L. (2003). Finger flexors fatigue in trained rock-climbers and untrained sedentary subjects. International fournal of Sport Medicine, 24, 424-427.

Schweizer, A. (2001). Biomechanical properties of the crimp grip position in rock climbers. Fournal of Biomechanics, 34, 217-223.

Sjogaard, G., Savard, G., \& Juel, C. (1988). Muscle blood flow during isometric activity and its relation to muscle fatigue. European fournal of Applied Physiology, 57, 327-335.

Usaj, A. (2002). Differences in the oxygenation of the forearm muscle during isometric contractions in trained and untrained subjects. Cellular and Molecular Biology Letters, 7, 375-377.
Watts, P. B., Dagget, M., Gallagher, P., \& Wilkins, B. (2000). Metabolic response during sport rock climbing and the effects of active versus passive recovery. International fournal of Sport Medicine, 21, 185-190.

Watts, P. B., \& Drobish, K. M. (1998). Physiological responses to simulated rock climbing at different angles. Medicine and Science in Sports and Exercise, 30, 1118-1122.

Watts, P. B., Newbury, V., \& Sulentic, J. (1996). Acute changes in handgrip strength, endurance, and blood lactate with sustained sport rock climbing. Fournal of Sports Medicine and Physical Fitness, 36, 255-260. 